\title{
Gliomatose Cerebral: Caso Clínico e Revisão da Literatura
}

\section{Gliomatosis Cerebri: Case Report and Literature Review}

Rui Ramos ${ }^{1}$, Cristiano Antunes ${ }^{1}$, Maria João Machado¹, Renata Marques ${ }^{1}$, Tiago Gil Oliveira², Mariana Cruz³, Marta Almeida4, Júlia Amorim ${ }^{4}$ Rui Almeida ${ }^{1}$

Autor Correspondente: Rui Ramos [ruimramos@hotmail.com] Rua das Sete Fontes, 4710-243 Braga, Portugal

\section{RESUMO}

Relatamos o caso de um doente com diagnóstico de gliomatose cerebral que após dois quadros de hipertensão intracraniana grave, iniciou quimioterapia paliativa e evoluiu de forma clínica e imagiologicamente satisfatória.

Doente de 33 anos de idade, previamente autónomo (Karnofsky Performance Status Scale de 100 pontos) e sem antecedentes pessoais/familiares relevantes. É investigado com tomografia computorizada e ressonância magnética cerebral no contexto de crise convulsiva inaugural. É detetada uma lesão infiltrativa fronto-parietal direita com extensão parietal contralateral através do esplénio do corpo caloso. Foi submetido a biópsia lesional por neuronavegação com diagnóstico histológico de astrocitoma grau II (OMS). Perante a integração imagiológica e anatomopatológica define-se então um processo de gliomatose cerebral. Após alta hospitalar recorre por duas vezes ao serviço de urgência com quadro de hipertensão intracraniana grave, uma das quais em estado de coma e com anisocoria pupilar. Após internamento e corticoterapia endovenosa em altas doses, recupera neurologicamente para o seu estado basal. Vinte e sete meses após o diagnóstico, encontra-se assintomático, sem défices neurológicos focais e com estabilidade imagiológica da lesão tumoral.

A gliomatose cerebral é uma neoplasia rara, de apresentação clínica variável e com um diagnóstico baseado nas características imagiológicas e histológicas. Após o início dos tratamentos complementares, nomeadamente quimioterapia, poderá ser possível uma estabilização clínica e imagiológica prolongada.

PALAVRAS-CHAVE: Neoplasias Cerebrais; Neoplasias Neuroepiteliais

\section{ABSTRACT}

We report a case of gliomatosis cerebri that after two episodes of severe intracranial hypertension, started palliative chemotherapy and progressed satisfactory clinically and imagiologically. A 33-year-old male patient, previously autonomous

1. Serviço de Neurocirurgia, Hospital de Braga, Braga, Portugal. 2. Serviço de Neurorradiologia, Hospital de Braga, Braga, Portugal. 3. Serviço de Anatomia Patológica, Hospital de Braga, Braga, Portugal. 4. Serviço de Oncologia Médica, Hospital de Braga, Braga, Portugal.

Recebido: 10/10/2016 - Aceite: 05/09/2017 
(Karnofsky Performance Status Scale of 100 points) and with no relevant personal/familiar history, is investigated with brain computed tomography and magnetic resonance imaging after an inaugural seizure. A right fronto-parietal infiltrative lesion with contralateral extension through the splenium of the corpus callosum was detected. A neuronavigation lesion biopsy was performed and the histological diagnosis revealed an astrocytoma grade II (WHO), defining a gliomatosis cerebri. After hospital discharge, he returns two times to the emergency department with severe intracranial hypertension. After hospitalization and intravenous corticosteroid therapy, he recovers neurologically to his basal state. Twenty-seven months after the diagnosis, the patient is asymptomatic, with no focal neurological deficits and with imaging stability of the lesion.

Gliomatosis cerebri is a rare neoplasm, with a variable clinical presentation and a diagnosis based on imaging and histological characteristics. After the start of the complementary treatments, such as chemotherapy, it is possible to get a long term clinical and imaging stabilization.

KEYWORDS: Brain Neoplasms; Neoplasms, Neuroepithelial

\section{INTRODUÇÃO}

A gliomatose cerebral é definida pela Organização Mundial de Saúde (OMS) como um processo infiltrativo glial com envolvimento inicial de mais de dois lobos cerebrais. ${ }^{1}$ Pode ser classificada em tipo I, ou clássico, em que não se individualiza uma massa tumoral, ou em tipo II, no qual está presente uma ligeira massa tumoral. ${ }^{2}$ Histologicamente caracteriza-se como uma neoplasia infiltrativa de astrócitos. ${ }^{2} \mathrm{Na}$ imuno-histoquímica, pode estar presente a mutação da isocitrato desidrogenase 1 (IDH 1) (comum nos astrocitomas de baixo grau e glioblastomas secundários), sendo mais frequente na gliomatose cerebral do tipo II. ${ }^{2}$ Afeta indivíduos de várias idades com um pico de incidência entre os 40-50 anos e um predomínio pelo sexo masculino. ${ }^{3}$ Perante a extensão inicial da doença, o papel do neurocirurgião resume-se habitualmente à realização de uma biópsia lesional. O tratamento envolve habitualmente quimioterapia, geralmente com temozolamida com diferentes esquemas de acordo com o centro oncológico. ${ }^{1}$ A realização de radioterapia não é consensual. ${ }^{4} \mathrm{O}$ fator mais relevante na determinação da sobrevivência é o grau histológico do tumor, sendo a sobrevivência média nos doentes submetidos a quimioterapia e/ou radioterapia de 26,6 meses, ${ }^{1}$ inferior à dos astrocitomas focais de grau sobreponível.

Este estudo descreve um caso clínico de gliomatose cerebral que após uma fase inicial de marcada deterioração neurológica, iniciou corticoterapia endovenosa e posteriormente quimioterapia com temozolamida, com recuperação neurológica total e prolongada estabilização clínica e imagiológica. Foi também efetuada uma revisão bibliográfica acerca do tema.

\section{CASO CLÍNICO}

Doente do sexo masculino de 33 anos, caucasiano, previamente autónomo (Karnofsky Performance Status Scale = 100), sem medicação habitual nem antecedentes pes- soais/familiares relevantes. Recorre ao serviço de urgência por crise convulsiva tónico-clónica generalizada com cerca de 5 minutos de duração e posterior recuperação espontânea do estado de consciência. É investigado com tomografia computorizada (TC) e ressonância magnética (RM) cerebral (Fig. 1) onde é detetada uma lesão fronto-parietal direita com envolvimento do esplénio do corpo caloso e extensão contralateral para o lobo parietal esquerdo. Foi submetido a biópsia lesional guiada por neuronavegação com diagnóstico histológico de astrocitoma difuso, grau II da OMS (não foi efetuada imuno-histoquímica) (Fig. 2). Não ocorreram complicações do procedimento cirúrgico. Atendendo à extensão da doença é decidido tratamento paliativo inicialmente com temozolamida ( $200 \mathrm{mg} / \mathrm{m}^{2}$ durante 5 dias de 28/28

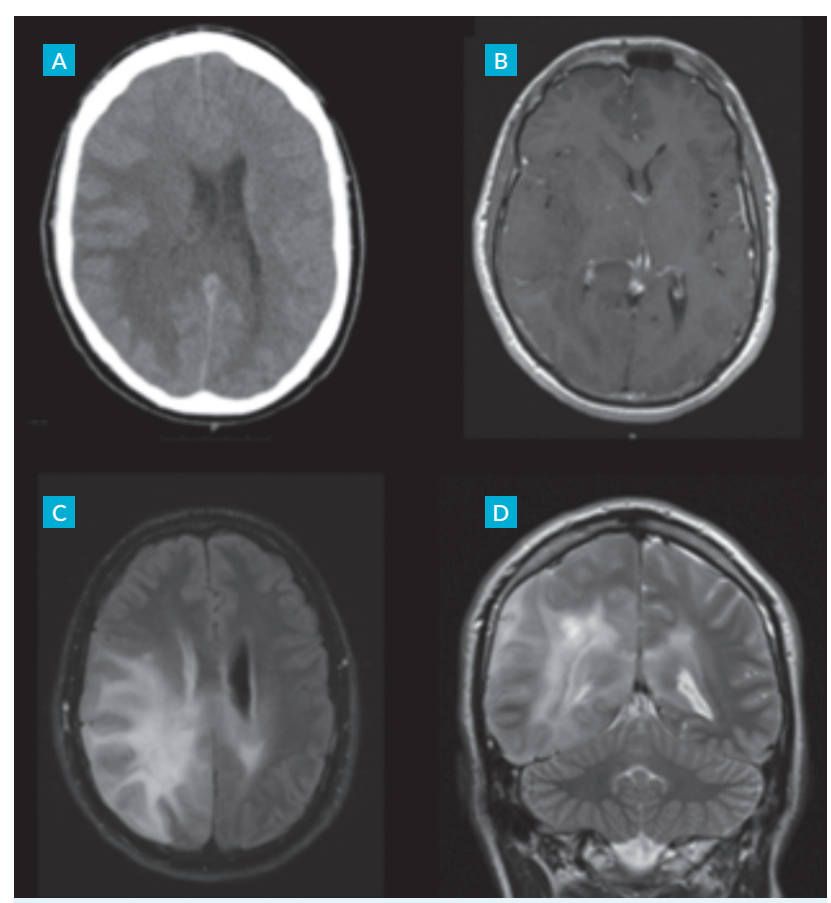

FIGURA 1. TC (A) e RM (B, C e D) cerebral pré-operatórias. É visível uma lesão intra-axial, cortico-subcortical, fronto-parietal direita. Estende-se à substância branca parietal do hemisfério contralateral, através do esplénio do corpo caloso. Apresenta hiposinal em T1 (B), hipersinal em FLAIR (C) e T2 (D) e não apresenta captação de contraste (B). 


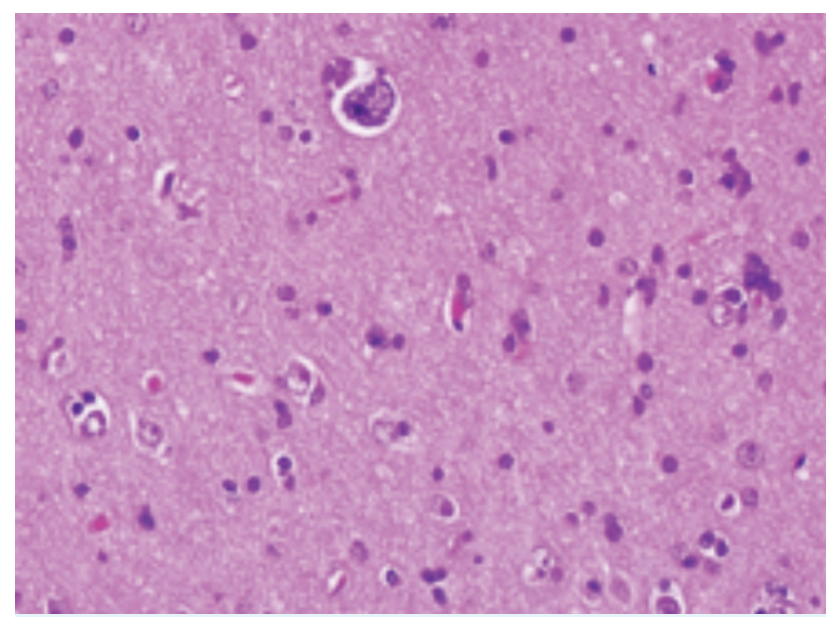

FIGURA 2. Histologia da biópsia lesional. A neoplasia é constituída maioritariamente por células de dimensões pequenas a intermédias, com núcleos arredondados e hipercromáticos. Corresponde a um astrocitoma grau II da OMS.

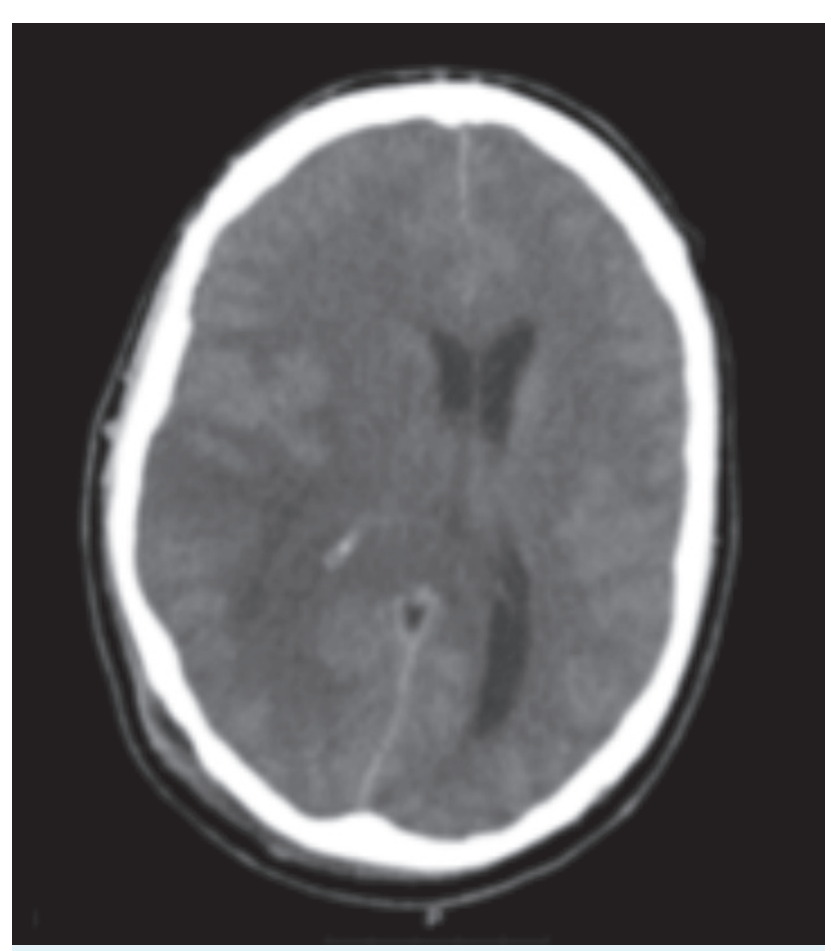

FIGURA 3. TC cranioencefálica no primeiro episódio de hipertensão intracraniana. Visualiza-se um aumento do efeito de massa da lesão tumoral, condicionando maior desvio das estruturas da linha média.

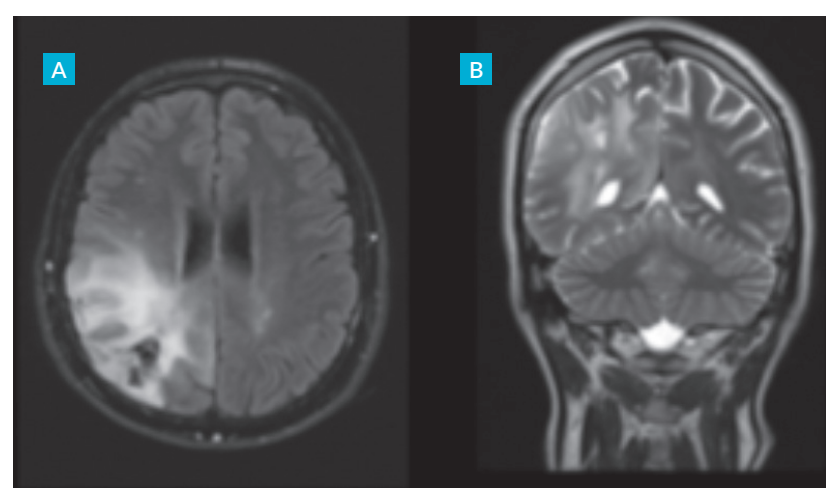

FIGURA 4. RM cerebral de controlo, cerca de 27 meses após o diagnóstico inicial. A - Corte axial em FLAIR. B - Corte coronal em T2. Verifica-se a infiltração tumoral de características e dimensões sobreponíveis à RM inicial. dias) e posterior reavaliação para decisão quanto à realização de radioterapia holocraniana. Dois dias após a biópsia, regressa ao serviço de urgência em coma, com resposta motora em descerebração, midríase à direita e exteriorização tumoral pela ferida cirúrgica. Repetiu a TC cranioencefálica (Fig. 3) onde foi evidente um aumento do efeito de massa da infiltração tumoral. Iniciou corticoterapia endovenosa com 10 mg de dexametasona de 8/8 horas, tendo recuperado para o seu estado neurológico habitual, sem défices neurológicos focais. Foi submetido a nova intervenção cirúrgica para encerramento da ferida cirúrgica e do orifício de trépano com colocação de uma rede de titânio. Um mês após a alta hospitalar, regressa novamente ao serviço de urgência com novo quadro de hipertensão intracraniana grave. Realizou novamente um ciclo de corticoterapia endovenosa com as mesmas doses, apresentando um marcado alívio sintomático. Teve alta com metilprednisolona oral 16 mg de 8/8 horas em esquema de desmame.

Iniciou então tratamento complementar com temozolamida (200 mg/m² durante 5 dias de 28/28 dias), tendo já realizado seis ciclos. Vinte e sete meses após o diagnóstico mantém-se assintomático (Karnofsky Performance Status Scale $=100$ ) e com estabilidade imagiológica da lesão (Fig. 4). Não apresentou evidência de toxicidade clínica nem hematológica à temozolamida.

\section{DISCUSSÃO}

Descrita pela primeira vez por Nevim em 1938, a gliomatose cerebral é uma neoplasia primária, infiltrativa, difusa e rara. $^{5}$ Geralmente estão envolvidos desde o diagnóstico mais de dois lobos cerebrais, podendo também ocorrer extensão para estruturas infratentoriais. ${ }^{5} \mathrm{~A}$ melhor característica que a diferencia de uma neoplasia glial clássica é a disparidade entre a quantidade de parênquima cerebral envolvido e os défices neurológicos focais do doente. ${ }^{6}$ Devido ao facto de se tratar de uma doença disseminada, existem duas teorias para a sua origem: transformação neoplásica simultânea em diferentes regiões do parênquima cerebral (hipótese oligoclonal) ou clone único de células com posterior disseminação multitópica (hipótese monoclonal). ${ }^{6}$

É mais comum entre a quarta e quinta décadas, ${ }^{5}$ e ocorre mais frequentemente no sexo masculino (ratio masculino:feminino de 19:6), tal como no caso do nosso doente. ${ }^{3}$ Poderá haver uma predisposição genética em doentes com doença de Von Recklinghausens. ${ }^{1}$

A apresentação clínica não é específica, podendo ocorrer cefaleias, paresia de pares cranianos, diplopia, amnésia, ataxia e crises convulsivas, sendo esta última a forma 
de apresentação clínica mais frequente. ${ }^{5}$ Quadros clínicos de hipertensão intracraniana são muito raros, ${ }^{5}$ mas o nosso paciente teve dois quadros graves, um dos quais com estado comatoso. Devido a apresentação clínica não ser específica, o diagnóstico diferencial é difícil e pode ocorrer uma suspeita inicial de uma lesão inflamatória ou infeciosa com consequente atraso no diagnóstico definitivo. ${ }^{5}$

A RM cerebral é o exame mais útil para o seu diagnóstico, apresentando-se como lesões com iso ou hiposinal em T1, hipersinal em T2, reduzido efeito de massa, ausência de captação de contraste e de restrição à difusão. A espetroscopia pode ser normal, mas geralmente apresenta uma ligeira elevação da colina e diminuição do $\mathrm{N}$-acetil-aspartato. $\bigcirc$ mioinositol pode estar elevado. ${ }^{5}$ A melhor ponderação para avaliar a extensão da doença são as ponderações em T2. ${ }^{3}$ Um achado relevante na RM é a infiltração difusa do córtex com alargamento dos sulcos corticais e reduzida diferenciação da substância branca e cinzenta. ${ }^{1}$ A chave do diagnóstico é um envolvimento difuso, contíguo, de predomínio central e com preservação da arquitetura das estruturas cerebrais. ${ }^{1}$ No caso do nosso doente as características imagiológicas são sobreponíveis às descritas, mas não foi realizada espetroscopia.

No diagnóstico diferencial devem-se considerar patologias como a encefalite vírica, encefalite tuberculosa e neurossarcoidose. ${ }^{7}$

Devido à ausência de correlação entre as características imagiológicas e a sobrevivência a obtenção de um diagnóstico histológico torna-se relevante para se estabelecer um prognóstico e decisão quanto a tratamentos complementares. A biópsia geralmente demonstra uma proliferação do componente astrocítico com preservação da arquitetura cerebral envolvente. A imuno-histoquímica geralmente é positiva para o p53 e WT1 na neoplasia glial. No nosso caso não foi realizado o estudo imuno-histoquímico.

Como se trata de uma doença difusa, uma craniotomia clássica geralmente não está indicada, exceto em casos de hipertensão intracraniana refratária ao tratamento médico. ${ }^{2,4}$ A abordagem padrão consiste na realização de uma biópsia lesional seguida de quimioterapia e/ou radioterapia. ${ }^{5} \mathrm{O}$ nosso paciente foi submetido a uma biópsia guiada por neuronavegação. $O$ primeiro quadro de hipertensão intracraniana provocou uma deiscência da ferida cirúrgica e exteriorização tumoral pelo orifício do trépano. Foi necessária a correção cirúrgica do orifício do trépano, mas o quadro de hipertensão intracraniana reverteu na totalidade com corticoterapia endovenosa. Segundo Vates et al, a realização de radioterapia como único tratamento complementar ou associada a quimioterapia permite um aumento da sobrevivência quando comparada com doentes que não realizaram qualquer tipo de tratamento. ${ }^{6}$ Contudo, os doentes que não foram submetidos a tratamento eram mais velhos e com valores de Karnofsky inferiores, pelo que estas conclusões têm um valor limitado.

A realização de radioterapia não é consensual. ${ }^{4}$ Devido à disseminação da doença seria mais indicado um regime de radioterapia holocraniana, o qual está associado a elevada morbilidade para o doente. A radioterapia pode ter algum benefício na progressão livre da doença, mas não tem benefício aparente na sobrevivência média.,8 Contudo, um estudo de Chen et al ${ }^{2}$ revelou uma associação entre a realização de radioterapia e sobrevivência, mas como se trata de um estudo retrospetivo esta associação é apenas especulativa. ${ }^{2}$ Segundo Landi et al, no protocolo para crianças com gliomatose cerebral, a presença de recrescimento tumoral ou agravamento neurológico é o fator decisivo para a realização de radioterapia holocraniana ou estereotáxica. ${ }^{1}$ Yerramneni et al, referem que se pode utilizar inicialmente temozolomida em monoterapia e reservar a radioterapia apenas quando for documentada progressão tumoral. ${ }^{4} \mathrm{~A}$ dose de radioterapia holocraniana poderá ser de 50 Gy, 54 ou 60 Gy em 30 frações ${ }^{4}$ mas não parece haver diferença na resposta clínica e imagiológica com doses > 55 Gy comparadas com doses < 54 Gy.

Existem diversos fármacos utilizados para quimioterapia na gliomatose cerebral e com impacto aparente na sobrevivência. Poderá ser utilizada temozolamida $75 \mathrm{mg} / \mathrm{m}^{2} /$ dia durante 21 dias em 12 ciclos de 28 dias, ${ }^{1}$ PCV (procarbazina $60 \mathrm{mg} / \mathrm{m}^{2}$, CCNU (lomustina) $110 \mathrm{mg} / \mathrm{m}^{2}$ e vincristina $\left.1,4 \mathrm{mg} / \mathrm{m}^{2}\right),{ }^{8}$ nitrosureias, ${ }^{2}$ cisplatina, nimustina ${ }^{9} \mathrm{e}$ BCNU (carmustina). ${ }^{7}$ Não existem diferenças significativas entre a temozolamida e PCV, apesar da temozolomida ser melhor tolerada e ser considerada a primeira linha de tratamento. ${ }^{8}$

futuro do tratamento para a gliomatose cerebral poderá passar por quimioterapia de acordo com a base genética específica do tumor. ${ }^{2}$ Não existe neste momento um protocolo de abordagem bem definido assim como consenso sobre o impacto da radioterapia e quimioterapia. $\bigcirc$ painel de peritos presente na reunião de gliomatose cerebral em Paris em 2015 recomenda após o diagnóstico inicial a realização de quimioterapia de acordo com os esquemas para os gliomas de alto grau e posterior realização de radioterapia após progressão tumoral. Tratamentos anti-angiogénicos, como bevacizumab aparentemente não têm eficácia comprovada, o que pode ser explicado devido à reduzida vascularização e ausên- 
cia de captação de contraste destes tumores. ${ }^{10}$ Contudo, recentemente Burger et al, referem que o bevacizumab poderá ser considerado em pacientes com gliomas de padrão maioritariamente infiltrativo. ${ }^{11}$

No nosso paciente foi decidido inicialmente a realização de quimioterapia isolada com temozolamida e posteriormente será reavaliado em consulta de grupo oncológico, sendo ponderada radioterapia holocraniana caso se documente progressão tumoral.

Diferentes fatores parecem ter influência na sobrevivência, tais como o grau histológico do tumor, a idade do paciente, o estado clínico prévio do paciente, ${ }^{5}$ o volume tumoral e a presença de mutação IDH1.7 Doentes com idade mais jovem têm habitualmente uma maior percentagem de mutações IDH 1, o que poderá justificar uma melhor sobrevivência.7 No caso de gliomatose cerebral com grau histológico II a sobrevivência média é de 34 meses, no grau III é de 15,5 meses e no grau IV é de apenas 8,5 meses. $^{2} \bigcirc$ facto da sobrevivência ser inferior à dos gliomas clássicos de grau histológico semelhante parece ser devido à impossibilidade de realização de uma craniotomia para citorredução tumoral significativa devido à sua marcada infiltração. ${ }^{2}$ O número de lobos cerebrais envolvidos não parece estar relacionado com a sobrevivência. ${ }^{2}$

A gliomatose cerebral é uma neoplasia rara, de apresentação clínica variável e com dois pilares de diagnóstico fundamentais: imagiológico e anatomopatológico.

Apesar do prognóstico sombrio e da rápida progressão na ausência de tratamento, o início de tratamentos complementares, nomeadamente quimioterapia, poderá permitir uma remissão sintomática e estabilização prolongada da doença.

CONFLITOS DE INTERESSE: Os autores declaram não ter qualquer conflito de interesse na realização do presente trabalho.

FONTES DE FINANCIAMENTO: Não houve qualquer fonte de financiamento na realização do presente trabalho.

CONFIDENCIALIDADE DOS DADOS: Os autores declaram ter seguido os protocolos da sua instituição acerca da publicação dos dados de doentes.

PROTEÇÃO DE PESSOAS E ANIMAIS: Os autores declaram que os procedimentos seguidos na elaboração do presente trabalho estão em conformidade com as normas das comissões de investigação clínica e de ética, bem como da declaração de Helsínquia e da Associação Médica Mundial.
CONFLICTS OF INTEREST: The authors declare that they have no conflicts of interest.

FINANCIAL SUPPORT: This work has not received any contribution, grant or scholarship.

CONFIDENTIALITY OF DATA: The authors declare that they have followed the protocols of their work center on the publication of data from patients.

PROTECTION OF HUMAN AND ANIMAL SUBJECTS: The authors declare that the procedures followed were in accordance with the regulations of the relevant clinical research ethics committee and with those of the Code of Ethics of the World Medical Association (Declaration of Helsinki).

\section{REFERÊNCIAS}

1. Landi A, Piccirilli M, Mancarella C, Giangaspero F, Salvati M. Gliomatosis cerebri in young patients' report of three cases and review of the literature. Childs Nerv Syst. 2011;27:19-25.

2. Chen S, Tanaka S, Giannini C, Morris J, Yan ES, Buckner J, et al. Gliomatosis cerebri: clinical characteristics, management, and outcomes. J Neurooncol. 2013;112:267-75.

3. Rajz GG, Nass D, Talianski E, Pfeffer R, Spiegelmann R, Cohen ZR. Presentation patterns and outcome of gliomatosis cerebri. Oncol Lett. 2012;3:209-13.

4. Yerramneni VK, Vinjamuri SR, Purohit AK, Sundaram C. Gliomatosis cerebri: Case series of six cases with review of literature. J Neurosci Rural Pract. 2015;6:602-6.

5. Blanco-Iglesias E, Cabeza B, Esparza EG, de Prada I, Lassaletta A, Budke M, et al. An Adolescent presenting with seizures as a symptom of gliomatosis cerebri. Pediatr Emerg Care. 2017 (in press).

6. Vates GE, Chang S, Lamborn KR, Prados M, Berger MS. Gliomatosis cerebri: a review of 22 cases. Neurosurgery. 2003;53:261-71.

7. Carroll KT, Hirshman B, Ali MA, Alattar AA, Brandel MG, Lochte B, et al. Management and survival patterns of patients with gliomatosis cerebri: A SEER-Based Analysis. World Neurosurg. 2017;103:186-93.

8. Lee HG, Lee KS, Lee WH, Kim ST. Procarbazine, CCNU, and vincristine chemotherapy in gliomatosis cerebri. Brain Tumor Res Treat. 2014;2:102-7.

9. Jung TY, Yoon MS, Kim YH, Jung S, Kim IY, Jang WY, et al. Gliomatosis cerebri having a poor performance status without recurrence after radiotherapy: a single institutional experience. Clin Neurol Neurosurg. 2015;130:1-5.

10. Greenfield JP, Castaneda Heredia A, George E, Kieran MW, Morales La Madrid A. Gliomatosis cerebri: A consensus summary report from the First International Gliomatosis cerebri Group Meeting, March 26-27, 2015, Paris, France. Pediatr Blood Cancer. 2016;63:2072-7.

11. Burger MC, Mildenberger IC, Wagner M, Mittelbronn M, Steinbach JP, Bahr O. Bevacizumab for patients with recurrent gliomas presenting with a gliomatosis cerebri growth pattern. Int J Mol Sci. 2017;18: E726. 